\section{Die Europäische Union als strategischer Akteur für globa- le Nachhaltigkeit}

\author{
von Hermann E. Ott und Min-ku Chung, \\ Wuppertal Institut für Klima, Umwelt, Energie \\ $\mathrm{GmbH}$
}

Der Artikel untersucht Wege, mit deren Hilfe die Europäische Union als Akteur auf der Weltbühne die Leitidee einer nachhaltigen Entwicklung auf globaler Ebene fördern kann. Denn die Union hat im EU-Vertrag das Ziel der nachhaltigen Entwicklung verankert und eine weit reichende, integrative Nachhaltigkeitsstrategie beschlossen. Auch in den Außenbeziehungen sollen die Prinzipien nachhaltiger Entwicklung befolgt und deren globale Umsetzung befördert werden. Dieses Ziel lässt sich nur erreichen, wenn die EU eine Strategie der ,politischen Globalisierung“ zur Gestaltung der weltweiten Wirtschaftsprozesse verfolgt.

Ausgehend von der Analyse der globalen Rahmenbedingungen für eine solche Strategie, die von einer zunehmenden ökonomischen, technologischen und kulturellen Globalisierung sowie der militärischen und ökonomischen Hegemonie der USA geprägt sind, werden in dem Beitrag die Möglichkeiten aufgezeigt, wie die Europäische Union ihre Vorstellungen von einer zukunftsfähigen Entwicklung auch unabhängig von den USA, die sich der Einbindung in völkerrechtliche Abkommen verweigert, verfolgen kann. Die immer weiter fortschreitende Vernetzung der wirtschaftlichen Akteure und Märkte erfordert eine ebenfalls wachsende Integration und Vernetzung der politischen Akteure. Dabei kann die EU im globalen politischen Mehrebenensystem von ihrer Erfahrung im Binnenraum profitieren, und mittels plurilateraler Diplomatie Europa als attraktives GegenModell zu dem ressourcenintensiven Modell der "alten“ Industriestaaten aufbauen und auf diesem Wege - unter Einbindung der relevanten Akteure der internationalen Zivilgesellschaft - kooperationswillige Staaten in ihre Strategie einbinden. Über die Einrichtung globaler Politiknetzwerke kann mit Hilfe einer flüssigen, nicht-hierarchischen Organisationsstruktur auf komplexe Probleme globalen Regierens unter den Bedingungen von Globalisierung und Hegemonie reagiert werden.

\section{Einleitung}

Nicht nur nationale Regierungen kämpfen mit den Herausforderungen einer ökonomisch, technologisch und kulturell globalisierten Welt. Selbst die Europäische Union, weltweit das Paradepferd wirtschaftlicher und zunehmend auch politischer Integration, muss sich mit veränderten globalen Strukturen und Verhältnissen auseinandersetzen. Auch der europäische Wirtschaftsraum steht unter Globalisierungs- bzw. Wettbewerbsdruck. Die Mitgliedstaaten der EU geben dementsprechend zunehmend einen Teil ihrer souveränen Befugnisse an die europäische Ebene ab und stärken somit die politischen Organe der Union. Ein ähnlicher Prozess auf globaler Ebene steht noch aus, denn die ökonomische und finanzielle Integration der Weltmärkte hat bisher noch kein politisches Pendant.

Die EU als politischer Akteur hat sich einer Strategie der globalen Nachhaltigkeit verpflichtet (Schepelmann 2001). Die Union hat nicht nur im EU-Vertrag das Ziel der nachhaltigen Entwicklung verankert (Artikel 2) und eine weit reichende, integrative Nachhaltigkeitsstrategie beschlossen (EU-Kommission 2001, sog. Lissabon-Strategie). Auch in den Außenbeziehungen sollen die Prinzipien nachhaltiger Entwicklung befolgt und deren globale Umsetzung befördert werden. Noch weiter gehend wird in der neuen EU-Verfassung der nachhaltigen Entwicklung ein äußerst prominenter Platz eingeräumt: Nach Artikel 3, der die Ziele definiert, soll die Union für eine nachhaltige Entwicklung Europas arbeiten und in ihren Außenbeziehungen beitragen zu Frieden, Sicherheit, einer nachhaltigen Entwicklung der Erde, Solidarität (...), freiem und fairem Handel, der Abschaffung von Armut und dem Schutz der Menschenrechte.

Dies impliziert eine zunehmende ,politische Globalisierung“ (Agarwal 2003, S. 231) zur Gestaltung der weltweiten Wirtschaftsprozesse, welche eine abgestimmte, in hohem Maße kooperative und transparente Politik erfordert. Gleichzeitig aber ist die weltpolitische Situation zu Beginn des 21. Jahrhunderts gekennzeichnet durch einen „unwilligen“ Hegemon, die Vereinigten Staaten, die verbindlichen Pflichten und multilateraler Einbindung ablehnend gegenüber stehen. Der Europäischen Union kommt in dieser Situation eine Schlüsselrolle $\mathrm{zu}$ - eine große Chance für das Hinauswachsen Europas hin zu 
einer verantwortlichen, kooperativen Rolle in der Weltpolitik (Wuppertal Institut 2005).

In den folgenden Ausführungen soll kurz ein Überblick über die politischen Rahmenbedingungen für eine globale Nachhaltigkeitspolitik seit Anfang der 90er Jahre gegeben werden. Sodann werden die Europäische Union als Akteur für eine nachhaltige Entwicklung skizziert und einige Handlungsmöglichkeiten für Allianzen mit staatlichen und nicht-staatlichen Akteuren vorgestellt.

\section{Globalisierung und Hegemonie}

Nach der jahrzehntelangen Eiszeit des „Kalten Krieges“ waren die 90er Jahre des letzten Jahrhunderts geprägt von einer Schwindel erregenden Beschleunigung der Verhältnisse. Das politische Tauwetter nach dem Fall des ,eisernen Vorhangs“ beschleunigte einen Prozess, den wir mit dem Begriff „Globalisierung“ kennzeichnen. In der Weltpolitik schien Anfang der 90er Jahre ebenfalls eine neue Zeit anzubrechen. Die von US-Präsident George Bush senior ausgerufene „Neue Weltordnung“ unter dem Dach der Vereinten Nationen war geprägt von der damals herrschenden Aufbruchstimmung nach Beendigung des Kalten Krieges. Die 90er Jahre waren im Gefolge des „Erdgipfels“ von Rio 1992 auch die Ära der Konferenzen über die großen Themen nachhaltiger Entwicklung, nämlich Umwelt und Menschenrechte: Bevölkerung, Nahrung, Frauen, Soziales und die Entwicklung der Städte (Fues, Hamm 2001). Dies war die Welt der globalen Konferenzen und „stakeholder“-Dialoge, wo multilateral, auf vielen Ebenen und im Prinzip ergebnisoffen die Probleme dieser Welt gelöst oder zumindest gründlich diskutiert werden konnten.

Für die Umwelt war mit dem Erdgipfel 1992 dennoch nicht etwa das ersehnte ,ökologische Zeitalter“ angebrochen (Weizsäcker 1992), sondern eine Zeit des gesteigerten Ressourcendrucks. Denn die Erhöhung der wirtschaftlichen Produktion ist heute immer noch fast durchgehend mit einem gesteigerten Ressourcenverbrauch verbunden (Bringezu 2004). Doch die Endlichkeit der Biosphäre setzt natürliche, physische Grenzen für die „Plünderung“ unseres Planeten (Gruhl 1978).

Zugleich waren die 90er Jahre schon gekennzeichnet durch die Ausnahmestellung der
USA und ihrem beginnenden „hegemonialen Unilateralismus“ (Habermas 2004). Bereits während der Regierung von Präsident Clinton von 1992 bis 2000 wurde mit Ausnahme der Klimarahmenkonvention kein einziger der wichtigen Umweltverträge von den Vereinigten Staaten ratifiziert - weder die Biodiversitätskonvention und deren Biosafety-Protokoll noch das Kyoto-Protokoll. Dies lag vor allem am amerikanischen Senat, der einer Ratifizierung mit Zweidrittel-Mehrheit zustimmen muss und auch heute noch von anti-ökologischen und nationalistischen Kräften beherrscht wird.

Mit der Wahl von George W. Bush zum Präsidenten wurden schon zuvor bestehende unilaterale Tendenzen der USA verfestigt, also eine auf einseitiges Vorgehen ausgerichtete Politik hoffähig (Rifkin 2004). Die zu Beginn der Präsidentschaft noch recht unbestimmte außenpolitische Haltung der Bush-Administration erhielt nach den Terroranschlägen des 11. September 2001 eine hegemoniale und unilaterale Richtung. In der nationalen Sicherheitsstrategie aus dem Jahre 2002 wird schließlich der Vormachtanspruch der USA postuliert, versehen mit der Ankündigung, diese Position gegen jede andere Macht $\mathrm{zu}$ verteidigen (US Security Strategy 2002).

Die Einschätzungen der Zukunftsaussichten für die „zur Weltmacht verdammten“ USA (Hacke 2001) sind unterschiedlich: Zum Teil wird angenommen, die Veränderungen zum Ende des letzten Jahrhunderts seien profunder Natur und die Hegemonie der USA werde auf unbestimmte Zeit Bestand haben (Emmott 2003), für andere dagegen ist das einseitige Vorgehen der USA, wie z. B. die militärischen Aktionen im Irak, bereits ein untrügliches Zeichen des Niedergangs dieser Weltmacht (Todd 2003). Eine Legitimitätskrise machen auch andere Autoren aus (Kupchan 2003) und beschwören die Gefahr einer „Überdehnung“ des USamerikanischen Führungsanspruchs aufgrund der Vernachlässigung sog. „weicher“ Faktoren, also etwa gemeinsamer Werte (Nye 2003).

Wie auch immer die Aussichten für eine langfristige, globale Hegemonie der USA eingeschätzt werden, für die nächsten Jahrzehnte werden die USA in der Welt militärisch, wirtschaftlich und weltpolitisch dominieren. Diese globale Ausnahmestellung ohne echten Konkurrenten ist unabhängig von der jeweiligen 
US-Regierung und ihrer politischen Ausrichtung (Rifkin 2004, S. 336). Sie beruht auf der technologischen, wirtschaftlichen und kulturellen Übermacht der Vereinigten Staaten (Habermas 2004, S. 182), der sich keine Regierung - auch keine gutwillige - entziehen kann. Unabhängig von der jeweiligen Regierung werden die USA in den nächsten Jahren deshalb nur wenig Neigung zeigen, sich verstärkt in multilaterale, also mehrseitige Prozesse und Organisationen einbinden zu lassen - im besten Falle wäre ein „Multilateralismus á la carte“ zu erwarten (so der ehemalige Leiter des Planungsstabs im State Department, Richard Haass, nach Dettke 2002, S. 25). Die USA werden demnach globale Allianzen mit wechselnden Staaten eingehen, auch solche mit Staaten der Europäischen Union oder von Fall zu Fall auch eine Koalition mit der Union selbst. Doch wird, selbst bei gutem Willen der Administration, eine Einbindung in verpflichtende multilaterale Abkommen die Ausnahme bleiben (Ott 2003).

Die Aussichten für eine auf Kooperation und Multilateralität gegründete „Weltinnenpolitik“ (Bundespräsident Horst Köhler) sind deshalb nur schwer abzuschätzen. Während einerseits die Welt enger zusammenrückt - so nach den Terroranschlägen vom 11. September 2001 oder der Flutkatastrophe in Südasien Ende 2004 - und sich ein immer engeres Netz diplomatischer Beziehungen über den Globus spannt, so ist andererseits mit der US-amerikanischen Verweigerung einer Einbindung in kollektive Verträge ein großes Hindernis für eine gemeinsame Lösung der Weltprobleme entstanden. Dies betrifft nicht nur den Bereich der Umwelt und Nachhaltigkeit, sondern auch die Friedensund Sicherheitspolitik, wie bei der Verweigerung des Beitritts zum Landminenvertrag oder dem Statut des Internationalen Strafgerichtshofs.

Gleichzeitig differenziert sich der Rest der Welt weiter aus. Große Teile im Süden unserer Erdkugel erscheinen nur verschwommen auf den Radarschirmen der Politik. Dies gilt insbesondere für das sub-saharische Afrika und Lateinamerika. Lediglich politische oder ökologische Katastrophen erhellen blitzlichtartig jene Weltregionen, die von den globalisierten Finanz-, Informations- und Warenströmen im Wesentlichen abgekoppelt sind. Ganz anders dagegen viele Staaten Ost- und Südostasiens, die als kleine oder große „Tiger“ von sich re- den machen. Südkorea, Taiwan, Malaysia und vor allem die Volksrepublik China glänzen mit jährlichen wirtschaftlichen Wachstumsraten von 6,3\% und 9,1\%(Südkorea 2002 und VR China 2003, vgl. http://www.auswaertigesamt.de/www/de/laenderinfos/index_html) und haben hinsichtlich ihres Bruttosozialprodukts und des Ausstoßes an klimaschädlichen Gasen häufig das Niveau der alten Industrienationen erreicht (Ott, Winkler, Brouns et al. 2004).

In 20 oder 30 Jahren wird sich China als neue Weltmacht neben die USA und vielleicht Europa stellen (Brzezinski 1999; Bender 2003). Entscheidend für das ökologische Gleichgewicht unseres Planeten wird daher die Frage sein, ob diese Staaten dem ressourcenintensiven Modell der „alten“ Industriestaaten nachfolgen oder aber neue Wege einer zukunftsfähigen Entwicklung gehen. Von großer Bedeutung ist daher, ob diese Staaten einen „amerikanischen“ oder einen „europäischen“ Entwicklungspfad wählen. Das wird seinerseits in hohem Maße davon abhängen, ob Europa diesen Staaten als attraktives Modell erscheint und ob die Union fähig zur Einbindung und Führung ist.

\section{Europa als Akteur für eine global nach- haltige Entwicklung}

Die Europäische Union zu Beginn des 21. Jahrhunderts ist von einem tief greifenden Zwiespalt geprägt: eine in technischer und ökonomischer Hinsicht immer stärker zusammenwachsende Staatengemeinschaft, der jedoch das politische Projekt abhanden gekommen scheint. Bildete die bilaterale Welt der Blockkonfrontation noch einen geschützten Raum für die Integration („Das Wir entsteht im Widerspruch“ - Régis Debray), so ist die Union nunmehr auf eine intrinsische, in ihr selbst liegende Motivation für ihre Weiterentwicklung verwiesen. Zu stark für ein verschämtes Heraustreten aus der Weltpolitik, jedoch auch zu schwach für eine Führungsrolle, changiert die Außenpolitik Europas zwischen Kraftmeierei und Abstinenz.

Welche Rolle also kann das „alte Europa“ in dem Bestreben zur Durchsetzung einer global nachhaltigen Entwicklung spielen? Unbestritten ist, dass die Durchsetzung von Themen auf der internationalen Ebene einen starken „Anwalt“ braucht - in der Sprache der Politikwissenschaftler „Vorreiter“ genannt 
(Young, Osherenko 1995). In unserem immer noch sehr an Staaten orientierten internationalen System ist ab einem bestimmten Zeitpunkt ein staatlicher Akteur erforderlich, der die Idee verficht, Mitstreiter gewinnt und Maßnahmen zur Regulierung anstößt (Beck 2002, S. 419).

Könnte Europa ein solcher Akteur sein? Der „ökonomische Riese und politische Zwerg“, wie die Europäische Union oft beschrieben worden ist, hat sich gerade wirtschaftlich vergrößert, mit einer Erweiterung um zehn auf insgesamt 25 Mitgliedstaaten. Der Prozess der Angleichung der Lebensverhältnisse in den neuen und alten EU-Staaten wird gewaltige finanzielle und personelle Ressourcen verschlingen und könnte durchaus zu einer neuen „Nach-innenGewandtheit“ führen. Auch die Wirkung auf die politische Entscheidungsfähigkeit kann im Moment noch nicht abgeschätzt werden - in jedem Fall ist die komplexere Struktur der Union kein Faktor, der die Vereinbarung und Einhaltung gemeinsamer außenpolitischer Leitlinien vereinfacht. Allerdings würde die Annahme der Europäischen Verfassung durch die Mitgliedstaaten der EU zu effektiveren Entscheidungsverfahren und zu einer größeren personellen Kontinuität führen (Schaik, Egenhofer 2003).

Paradoxerweise mag es gerade der innere Problemdruck sein, der eine Vorreiterrolle der Europäischen Union für globale Ressourcengerechtigkeit eher wahrscheinlich macht. Denn die europäische Identität entsteht nicht aus dem gemeinsamen Wirtschaftsraum, nicht aus der gemeinsamen Währung und auch nicht aus der Vielfalt der europäischen Kulturen. Der $\mathrm{Zu}-$ sammenschluss Europas beruht auf einer Idee, die größer ist als der geographische Raum. Jean Monnet, der geistige Vater eines geeinten Europa, war nicht zuletzt beseelt durch den Wunsch, einen Beitrag zu einer besseren Welt zu leisten (,une contribution à un monde meilleur“).

Nur in Europa hat sich die Idee des Sozialstaats als gesellschaftliche Maxime durchgesetzt (Rifkin 2004). In Europa hat das Prinzip der Integration, des friedlichen Miteinander und der multilateralen Kooperation nach Jahrhunderten blutiger Auseinandersetzungen seinen Ausdruck gefunden. Diese Werte müssen sich zunächst in dem allmählichen Zusammenwachsen der größeren Union bewähren. Doch angesichts der drohenden globalen Gefahren können diese Werte nicht an den Grenzen des Kontinents halt machen. Erst die globale Durchsetzung europäischer Vorstellungen vom Zusammenleben der Völker erst wird auch für Europa jenes Klima schaffen, in dem Vielfalt, soziale Gerechtigkeit und die Freiheit des Einzelnen gedeihen können. Hier kann Europa auch seine ganz spezifischen Erfahrungen der Vielfalt in der Einheit einbringen - nicht als Aufhebung aller kulturellen und sozialen Differenzen, sondern als ein Miteinander (sich) selbst bewusster Kulturen. Ähnlich könnte ein kosmopolitisches Modell für eine faire und ressourcengerechte Welt aussehen. Mit den Worten von Ulrich Beck: Nicht in der Nabelschau, in der kosmopolitischen Öffnung Europas liegt die Lösung der Probleme (Beck 2004).

So haben die europäischen Staaten in den letzten 50 Jahren ein System der sich verdichtenden Kooperation untereinander geschaffen (Nuscheler 2001; Hamm, Hippler, Messner, Weller 2002). Europa verfügt daher über einen reichhaltigen Erfahrungsschatz an Mechanismen und Institutionen der Zusammenarbeit. Die Crux liegt, wie so häufig, in der Umsetzung. Der letzte Bericht der Kommission über die Ergebnisse der Nachhaltigkeitsstrategie konstatiert erhebliche Schwierigkeiten bei der Durchsetzung, die den gesamten Prozess gefährden können (EU-Kommission 2004). Andere Urteile sind noch strenger und bescheinigen der EU das Fehlen eines konsistenten Ansatzes sowie der Strategie nur eine sehr geringe Wirkung auf politische Prozesse (EEB 2004).

Andererseits tritt die Union in der Mehrzahl der Umweltverhandlungen tatsächlich als Motor eines vorsorgenden Umweltschutzes auf. In den Klimaverhandlungen ist die EU seit dem Inkrafttreten der Klimarahmenkonvention 1994 die treibende Kraft, häufig im Zusammenwirken mit Schlüsselstaaten aus der dritten Welt. (Oberthür, Ott 2000; Ott 2001). Ähnliches gilt für die Entwicklungspolitik. Zwar hinkt auch in diesem Bereich die Wirklichkeit der Rhetorik ein gutes Stück hinterher. Doch betreibt die Union schon seit Jahrzehnten eine Politik der bevorzugten Behandlung von Entwicklungsländern und hat über das Lomé-Abkommen einen Mechanismus geschaffen, mit nunmehr über 90 Staaten Afrikas, der Karibik und des pazifischen Raumes in der Wirtschaftspolitik in besonderer Weise zusammenzuarbeiten. Doch wird diese in Ansätzen gute Politik gegenüber dem Süden 
durch die hohen Agrarsubventionen wieder konterkariert, da sie die Entwicklung einer autarken Landwirtschaft im Süden verhindern.

Auch in der internationalen Handelspolitik ist die Union nicht ohne Fehl und Tadel. Gerade aus der Sicht von Entwicklungsländern erscheint die EU häufig als Zwillingsschwester der USA, wenn es um die Durchsetzung wirtschaftlicher Interessen geht. Der erzwungene Abbruch der WTO-Verhandlungen in Cancun durch eine Gruppe von wichtigen Entwicklungsländern richtete sich ebenso sehr gegen die einseitige Politik Europas wie gegen die USA (Narain 2003). In ökonomischer Hinsicht agiert Europa selbst in hohem Maße hegemonial und spricht des Öfteren der eigenen Rhetorik Hohn.

Allerdings scheint Europa lernfähiger zu sein. Der Einsatz für einen effektiven Klimaschutz ist nicht nur das Ergebnis einer vorsorgenden Politik für die eigene Bevölkerung, sondern entspringt ebenso sehr einer angenommenen Verantwortung für jene Weltgegenden, die besonders vom Klimawandel betroffen sein werden. Auch die Vorstellung einer „Festung Europa“, abgeschottet gegen wirtschaftliche und ökologische Flüchtlinge, erscheint den meisten Europäern unerträglich.

Für die in der EU zusammengeschlossenen Staaten bedeutet diese Analyse, Integration und Vertiefung zu beschleunigen, um als eigenständiger Akteur auf die Weltbühne zu treten. Dies hätte zur Folge, die Union vom bloßen Objekt der Globalisierungstendenzen (und im besten Fall Juniorpartner der USA) zu einem handelnden Subjekt der Geschichte zu entwickeln. Als „kooperative Weltmacht“ (Messner 2001) mit einer rationalen Außenpolitik hätte die Europäische Union die Chance, die globalen Bedingungen für ein friedliches Miteinander zu gestalten. Nach innen würde eine solche Vision Identität stiftend wirken und die sanfte Integration der neuen Mitglieder fördern. Inhaltlich ist allerdings Voraussetzung, dass die EU in weit stärkerem Maße konkrete Vorstellungen über eine nachhaltige Welt(-ordnung) entwickelt.

Eine wesentliche Grundlage für den Erfolg eines so komplexen Vorhabens ist die strikte Einhaltung des Prinzips der Rechtsgemeinschaft im Sinne Walter Hallsteins (Pernice 2003). Die EU kann in dieser Hinsicht auch als „Vorläuferin“ zukünftiger regionaler und globaler $\mathrm{Zu}$ sammenschlüsse verstanden werden: Bei einer großen Vielzahl an Akteuren und Regelungsebenen ist eine „Verrechtlichung“ der Beziehungen die einzige Möglichkeit, Willkür, Intransparenz und Ineffektivität zu vermeiden. Unvermeidlich auftretende Konflikte, so die Lehre aus der europäischen Geschichte, lassen sich am Besten auf einer über-parteilichen, juristischen Ebene entscheiden, mit einem höchsten Gericht als Garant von Menschenrechten, politischer Teilhabe, Abgrenzung von Kompetenzbereichen und der Durchsetzung politisch getroffener Regelungen.

\section{Strategische Allianzen für globale Nach- haltigkeit}

Eine europäische Strategie für globale Ressourcengerechtigkeit lässt sich nicht im Alleingang bewältigen, sondern braucht Partner und Verbündete. Europa muss daher nicht nur inhaltliche Vorstellungen einer gerechten und menschenwürdigen Welt gewinnen, sondern auch einen Plan für die außenpolitische Durchsetzung einer solchen Strategie entwickeln. Eine solche Strategie kann auf Allianzen mit ganz unterschiedlichen Partnern setzen, denn immer mehr Akteure betreten die internationale Bühne.

Auf diese Situation ist die Union im Grunde gut eingestellt, denn sie entspricht ziemlich genau der internen Struktur Europas: Brüssel und Straßburg sind die Zentren einer politischen Kultur, in der auf vielen Ebenen (lokal, regional, national, europäisch) und von einer ungeheuren Vielzahl an Akteuren in einem fast schon undurchschaubaren System Politik gemacht wird. Dieses so genannte „Mehrebenensystem“ der EU, wie es von Politikwissenschaftlern bezeichnet wird, funktioniert nach gänzlich anderen Regeln als die traditionelle Diplomatie (Scharpf 2002). In den sich herausbildenden vernetzten Politiksystemen geht es um Transparenz von Prozessen der Entscheidungsfindung, um die Teilhabe und Einbindung möglichst vieler Betroffener und um „Empathie“, also eine gesteigerte Fähigkeit zum Sich-Einfühlen in die Lage anderer (Rifkin 2004).

Eine wirksame Strategie zur Förderung einer global nachhaltigen Entwicklung in diesem Mehrebenensystem ist die Allianzenbildung oder plurilaterale Diplomatie. Vielfach wird aus einem falsch verstandenen Universalismus (,globale Probleme müssen global angepackt 
werden“) die Lösung der großen Probleme alleine in der UN-Diplomatie gesehen. Doch das kann zu großen zeitlichen Verzögerungen führen - vor allem dann, wenn ein wichtiger Akteur (wie die USA) sich nicht einbinden lassen will. In diesen Fällen muss nicht auf das langsamste Schiff gewartet werden, sondern es kann eine Strategie des „Universalismus minus $X^{\text {“ ver- }}$ folgt werden (Wuppertal Institut 2005). Beispiele für erfolgreiche plurilaterale Diplomatie lassen sich in der jüngsten Vergangenheit finden. So wäre weder die Landminenkonvention zum Verbot von Anti-Personenminen noch die Errichtung des Internationalen Strafgerichtshofs möglich gewesen, wenn Europa und Kanada auf die Bremser - vor allem auf die USA - gewartet hätten. Sehr häufig gehen plurilaterale Abkommen auch einer globalen Lösung voraus - die „like-minded-countries“ handeln hier als Vorreiter einer zukünftigen universellen Regelung.

Ein lohnenswertes Projekt für ein plurilaterales Abkommen wäre zum Beispiel die Einrichtung einer internationalen Organisation zur Förderung erneuerbarer Energien. Dazu bräuchte es nicht mehr als eine Hand voll Industrieländer, die einen Teil der finanziellen Ressourcen umleiten, die jetzt noch an die Internationale Energieagentur (IEA) oder die Internationale Atomenergiebehörde (IAEO) fließen. Von Eurosolar und dem Weltrat für erneuerbare Energien ist bereits das Statut einer International Renewable Energy Agency (IRENA) ausgearbeitet worden, also einer Internationalen Behörde für erneuerbare Energien (Scheer 2003). Der Bundestag hat im April 2003 die Gründung einer solchen Organisation befürwortet. Leider wurde jedoch die von Deutschland ausgerichtete Konferenz über erneuerbare Energien im Juni 2004 (http://www.renewables2004.de) nicht genutzt, um die Sache voranzubringen - hauptsächlich deshalb, weil die USA und Saudi Arabien in die Abschlusserklärung und den Nachfolgeprozess mit einbezogen werden sollten.

Im internationalen Mehrebenensystem des 21. Jahrhunderts sind die möglichen Partner für die Durchsetzung strategischer Ziele allerdings nicht mehr auf Staaten beschränkt. Neue Akteure der Zivilgesellschaft haben in den letzten Jahrzehnten viele Regionen internationaler Politik und Diplomatie erobert, vor allem im Bereich des Sozialen und der Ökologie. Für individuelle Akteure, Verbände und sozial motivierte Gruppen ist der transnationale Raum so zum Aktionsfeld geworden (Messner, Nuscheler 1996).

Eine europäische Strategie zur Durchsetzung globaler Ressourcengerechtigkeit und Nachhaltigkeit erfordert deshalb eine Allianz mit zumindest den großen Akteuren der Zivilgesellschaft aus Nord und Süd, wie zum Beispiel Oxfam, Greenpeace, WWF, Human Rights Watch, Amnesty International und dem Third World Network. Eine der einfachsten Unterstützungsleistungen wäre die Hilfe bei der Netzwerkbildung. So ist das Climate Action Network (CAN), welches ca. 300 Umweltorganisationen für den Klimaschutz umfasst, 1989 in Loccum mit Hilfe Deutschlands und Schwedens gegründet worden (Waddell 2003). Ohne CAN würde es in dieser Form weder die Klimarahmenkonvention noch das Kyoto-Protokoll geben (Oberthür, Ott 2000).

Es gibt ferner Beispiele für enge Allianzen zwischen Staaten und Organisationen der Zivilgesellschaft, die für eine europäische Strategie globaler Ressourcengerechtigkeit Vorbild sein könnten. Denn sowohl der Internationale Strafgerichtshof (http://www.iccnow.org/) wie auch die internationale Konvention gegen Anti-Personenminen (http://www.icbl.org/info/ history.html) wären nicht, oder nur in sehr geschwächter Form, zustande gekommen ohne die massive Unterstützung durch zivilgesellschaftliche Organisationen und deren Bündnis mit „wohlmeinenden“ Staaten. Da in beiden Fällen der jeweilige internationale Vertrag gegen den Widerstand der USA zustande gekommen ist, können sie auch als Vorbilder für eine anti-hegemoniale Strategie von Mittelund Großmächten gelten.

Eine noch weitergehende Form der Kooperation zwischen NGOs und Staaten ist die Bildung „hybrider“ Organisationen, die weder NGOs, also Nicht-Regierungsorganisationen, noch zwischenstaatliche Einrichtungen sind. Die bisherigen Foren der Zusammenkunft von Staaten und zivilgesellschaftlichen Akteuren sind entweder diplomatische Konferenzen und daher bestimmt durch die absolute Dominanz staatlicher Akteure, wobei die Zivilgesellschaft eher „schmückendes Beiwerk“ bildet. Oder sie sind ausgerichtet von nichtstaatlichen Akteuren, wie z. B. das Weltsozialforum 2000 in Porto Alegre mit vielen tausend Teilnehmern, 
auf denen Staatenvertreter lediglich in privater Funktion auftreten.

Eine hybride Organisation ist auf der annähernden Gleichheit ihrer Mitglieder aufgebaut, unterscheidet sich also stark von diplomatischen Konferenzen und solchen der Zivilgesellschaft. Ein Beispiel für eine solche hybride Organisation ist die International Union for the Conservation of Nature (IUCN, http://www.iucn.org/). Eine erfolgreiche Übertragung und Weiterentwicklung des IUCN-Modells hat schon einmal stattgefunden, nämlich bei der Errichtung der World Commission on Dams (http://www.dams.org). Die Kommission erarbeitete in einem mehrjährigen Prozess Vorschläge für ökologische und soziale Standards bei der Errichtung großer Staudämme. Diese Arbeit und Vorgehensweise gilt seither als ein wichtiges Beispiel für die Lösung internationaler Probleme durch „globale PolitikNetzwerke“: Über die Einbeziehung möglichst aller für ein globales Problemfeld wichtigen Akteure - Staaten, Unternehmen und Vertreter der Zivilgesellschaft - wird eine für alle Parteien annehmbare Regelung erarbeitet (vgl. Reinicke u. a. 2000; Streck 2002). So kann mit Hilfe einer flüssigen, nicht-hierarchischen Organisationsstruktur auf komplexe Probleme globalen Regierens reagiert werden.

\section{Fazit}

Eine der größten Herausforderungen des 21. Jahrhunderts wird darin bestehen, der zunehmenden ökonomischen und technologischen Globalität eine „politische Globalisierung“ zur Seite zu stellen. Es müssen also internationale Strukturen und Normen entwickelt werden, die den machtpolitischen Gegebenheiten Rechnung tragen und dennoch kooperativ, transparent und vor allem fair sind (Wuppertal Institut 2005). Die Europäische Union als Akteur hat sich die Förderung einer nachhaltigen und fairen Weltgesellschaft auf die Fahnen geschrieben. Bei dem Versuch einer Umsetzung dieser Strategie ergeben sich nicht nur EU-interne Widersprüche und Widerstände, sondern die Union sieht sich auch mit einer immer komplexer werdenden globalen politischen Ordnung konfrontiert.

Doch die Europäische Union ist selbst ein komplexes Mehrebenensystem mit harten und weichen Entscheidungsstrukturen. Dies kann dazu beitragen, das globale System besser zu verstehen und für die eigenen Zwecke zu nutzen. Auch die relativ einseitige, auf die Verfolgung nationaler Vorteile ausgerichtete Politik der jetzigen US-Administration muss dabei kein Hindernis sein. Längst sind Techniken der Allianzenbildung und der verrechtlichten Diplomatie (Ott 1998) entwickelt worden, die einen Umgang mit dem Hegemon erleichtern. In Zukunft wird es für die Union darauf ankommen, ein ausreichendes Selbstbewusstsein zu entwickeln, um für eine globale Führung gerüstet zu sein. An Motivation dürfte es nicht fehlen, denn - und damit soll noch einmal an Jean Monnet erinnert werden - das geeinte Europa sollte nichts weniger leisten als: une contribution à un monde meilleur.

\section{Literatur}

Agarwal, A., 2003: Globalization, Civil Society and Governance: Challenges for the 21st Century. In: Cross, N. (ed.): Evidence of Hope: The Search for Sustainable Development. London: Earthscan, S. 223-234

Beck, U., 2002: Macht und Gegenmacht im globalen Zeitalter. Frankfurt am Main: Suhrkamp

Beck, U., 2004: Der kosmopolitische Blick oder: Krieg ist Frieden. Frankfurt: Suhrkamp (Edition Zweite Moderne)

Bender, P., 2003: Weltmacht Amerika - Das Neue Rom. Stuttgart: Klett-Cotta Verlag

Bringezu, S., 2004: Erdlandung. Navigation zu den Ressourcen der Zukunft. Stuttgart, Leipzig: S. Hirzel-Verlag

Brzezinski, Z., 1999: Die einzige Weltmacht. Amerikas Strategie der Vorherrschaft. Frankfurt: Fischer Taschenbuch Verlag

Dettke, D., 2002: Das neue Interesse des Hegemonen an Verbündeten. In: Internationale Politik und Gesellschaft 1, S. 22-26

EEB - European Environmental Bureau, 2004: Towards an EEB Review of the EU Sustainable Development Strategy. Draft Version, Brüssel, 13. April 2004; http://www.eeb.org

Emmott, B., 2003: Vision 20/21. Die Weltordnung des 21. Jahrhunderts. Frankfurt: S. Fischer Verlag

EU-Kommission, 2001: Mitteilung der Kommission - Nachhaltige Entwicklung in Europa für eine bessere Welt: Strategie der Europäischen Union für die nachhaltige Entwicklung. Brüssel, den 15.5.2001, KOM(2001)264 endg. 
EU-Kommission, 2004: Die Lissabon-Strategie realisieren. Reformen für die Erweiterte Union Bericht der Kommission für die Frühjahrstagung des Europäischen Rates. Brüssel, den 20.2.2004, KOM (2004) 29 endg./2

Fues, T.; Hamm, B., 2001: Die Weltkonferenzen der 90er Jahre: Baustellen für Global Governance. Bonn: Dietz Verlag

Gruhl, H., 1978: Ein Planet wird geplündert. Die Schreckensbilanz unserer Politik. Frankfurt: S. Fischer Verlag

Habermas, J., 2004: Wege aus der Weltunordnung. In: Blätter für deutsche und internationale Politik Nr. 1, S. 27-45

Hacke, Ch., 2001: Zur Weltmacht verdammt. Die amerikanische Außenpolitik von J.F. Kennedy bis G.W. Bush. Berlin: Propyläen Verlag

Hamm, B.; Hippler, J.; Messner, D.; Weller, Ch., 2002: Weltpolitik am Scheideweg. Der 11. September 2001 und seine Folgen. SEF-Policy Paper 19, März 2002. Bonn: Stiftung Entwicklung und Frieden Kupchan, Ch.A., 2003.: The Rise of Europe, America's Changing Internationalism, and the End of U.S. Primacy. In: Political Science Quarterly 118:2, S. 205-231

Messner, D., 2001: Kooperative Weltmacht. Die Zukunft der Europäischen Union in der neuen Weltpolitik. In: Politik und Gesellschaft 1, S. 23-39

Messner, D.; Nuscheler, F. (Hrsg.), 1996: Weltkonferenzen und Weltberichte. Ein Wegweiser durch die internationale Diskussion. Stiftung Entwicklung und Frieden. Bonn: Verlag J.H.W. Dietz Nachfolger Narain, S., 2004: Sublimating Climate Change. In: Down to Earth, January 15; http://www.downtoearth.org.in

Nuscheler, F., 2001: Multilateralismus vs. Unilateralismus. Kooperation vs. Hegemonie in den transatlantischen Beziehungen. Stiftung Entwicklung und Frieden. Policy Paper 16 (Januar), Bonn

Nye, J.S., 2003: Das Paradox der amerikanischen Macht. Warum die einzige Supermacht der Welt Verbündete braucht. Hamburg: Europäische Verlagsanstalt

Oberthür, S.; Ott, H.E., 2000: Das Kyoto-Protokoll. Internationale Klimapolitik für das 21. Jahrhundert. Opladen: Leske + Budrich

Ott, H.E., 1998: Umweltregime im Völkerrecht. Eine Untersuchung über neue Formen internationaler institutionalisierter Kooperation am Beispiel der Verträge zum Schutz der Ozonschicht und zur Kontrolle grenzüberschreitender Abfallverbringungen. Baden-Baden: Nomos Verlag
Ott, H.E., 2001: Climate Change: An Important Foreign Policy Issue. In: International Affairs 77/2, S. 277-296

Ott, H.E., 2003: Umweltpolitik im Zeitalter der Hegemonie. In: Engelhardt, M.; Steigenberger, M. (Hrsg.): Die Umwelt in der Globalisierungsfalle. Hamburg: VSA-Verlag, S. 160-164

Ott, H.E.; Winkler, H.; Brouns, B.; Kartha, S.; Mace, M.J.; Huq, S.; Kameyama, Y.; Sari, A.P.; Pan, J.; Sokona, Y.; Bhandari, P.M.; Kassenberg, A.; La Rovere, E.L.; Rahman, A., 2004: South-North Dialogue on Equity in the Greenhouse. A proposal for an adequate and equitable global climate agreement. GTZ Climate Protection Programme, May 2004. Eschborn: Deutsche Gesellschaft für Technische Zusammenarbeit

Pernice, I., 2003: Begründung und Konsolidierung der Europäischen Gemeinschaft als Rechtsgemeinschaft. In: Zuleeg, M. (Hrsg.): Der Beitrag Walter Hallsteins zur Zukunft Europas. Referate zu Ehren von Walter Hallstein. Baden-Baden: Nomos, S. 56-70

Reinicke, W.H.; Deng, F.; Witte, J.M.; Benner, T., 2000: Critical Choices. The United Nations, Networks, and the Future of Global Governance. Ottawa: IDRC Publishers

Rifkin, J., 2004: Der Europäische Traum. Die Vision einer leisen Supermacht. Frankfurt, New York: Campus Verlag

Rosenau, J., 2002: Governance in a New Global Order. In: Held, D.; McGrew, A.: Governing Globalization. Power, Authority and Global Governance. Cambridge: Polity Press

Santarius, T.; Dalkmann, H.; Steigenberger, M.; Vorgelpohl, K., 2003: Grüne Grenzen für den Welthandel. Eine ökologische Reform der WTO als Herausforderung an eine Sustainable Global Governance. Wuppertal Paper Nr. 133. Wuppertal

Schaik, L. van; Egenhofer, C., 2003: Reform of the EU Institutions: Implications for the EU's Performance in Climate Negotiations. CEPS Policy Brief No. 40, Brussels: Centre for European Policy Studies; http://www.ceps.be/

Scharpf, F.W., 2002: Regieren im europäischen Mehrebenensystem. Ansätze zu einer Theorie. In: Leviathan 30/1, S. 65-92

Scheer, H., 2003: IRENA - Ein Weltprojekt im Spannungsfeld zwischen Uni- und Multilateralismus. In: Solarzeitalter 15/2, S. 2-4

Schepelmann, P., 2001: Kollaps oder Rückkehr zur Politik? Auf dem Weg zu einer Nachhaltigkeitsstrategie der Europäischen Union. In: Ökologisches Wirtschaften 2, S. 5-6 
Streck, Ch., 2002: Global Public Policy Networks as Coalitions for Change. In: Esty, D.; Ivanova, M. (eds.): Global Environmental Governance, Options and Opportunities. Yale School of Forestry and Environmental Studies, New Haven, Ct.

Todd, E., 2003: Weltmacht USA. Ein Nachruf. München: Piper Verlag

US Security Strategy, 2002: The National Security Strategy of the United States of America. Washington, September 2002; download unter http://www.whitehouse.gov

Waddell, S., 2005: The Climate Action Network: Civil Society Tackling Global Negotiations; download unter http://www.gan-net.net/about/examples.html Weizsäcker, E.U. von, 1992: Erdpolitik. Ökologische Realpolitik an der Schwelle zum Jahrhundert der Umwelt. Darmstadt : Wissenschaftliche Buchgesellschaft

Wuppertal Institut (Hrsg.), 2005: Fair Future. Begrenzte Ressourcen und globale Gerechtigkeit. München: C. H. Beck

Young, O.R.; Osherenko, G., 1995: Testing Theories of Regime Formation: Findings from a Large Collaborative Research Project. In: Rittberger, V. (ed.): Regime Theory and International Relations. Oxford: Clarendon Press, S. 223-251

\section{Kontakt}

Hermann E. Ott / Min-ku Chung

Wuppertal Institut für Klima, Umwelt, Energie

$\mathrm{GmbH}$

Hackesche Höfe

Rosenthaler Strasse 40/41, 10178 Berlin

Tel.: +49 (0) 30 / 2809 - 5489 (Sekr. -54 94)

Fax: +49 (0) 30 / 2809 - 4895

E-Mail: Hermann.Ott@wupperinst.org

Minku.Chung@wupperinst.org

Internet: http://www.wupperinst.org/berlin-office/ 